\title{
Association between Serum Uric Acid to HDL- cholesterol Ratio and Nonalcoholic Fatty Liver Disease among Chinese Adults
}

Hui Zhao

Qingdao University

Xia Qiu

Qingdao Bright moon Seaweed Group Co. Ltd

Jiajia Cui

Qingdao University

Yongye Sun ( $\square$ yongye.sun@126.com )

Qingdao University

\section{Research Article}

Keywords: Serum Uric Acid , HDL-cholesterol Ratio, Nonalcoholic Fatty Liver Disease, Chinese Adults

Posted Date: November 2nd, 2021

DOI: https://doi.org/10.21203/rs.3.rs-1025949/v1

License: (c) (i) This work is licensed under a Creative Commons Attribution 4.0 International License. Read Full License 
1 Association between Serum Uric Acid to HDL-cholesterol Ratio and

2 Nonalcoholic Fatty Liver Disease among Chinese Adults

${ }^{1}$ Department of Nutrition and Food Hygiene, School of Public Health, Qingdao University, Qingdao, China.

${ }^{2}$ State Key Laboratory of Bioactive Seaweed Substances, Qingdao Bright moon Seaweed Group Co. Ltd. Qingdao 266400, Shandong, China

E-mail of Hui Zhao: Alisazh7916@163.com;

E-mail of Xia Qiu: xia.qiu@ @msg.com;

E-mail of Jiajia Cui: 2570418819@qq.com;

Corresponding author: Dr Yongye Sun, Department of Nutrition and Food Hygiene, School of Public Health, Qingdao University, Qingdao 266021, China.

Tel:+86-138-6398-0712; Fax: 0532-83812434; Email: yongye.sun@126.com

\section{ABSTRACT}

We conducted this case-control study to explore the association of serum uric acid (SUA) to HDLcholesterol (HDL-C) ratio (UHR) with the risk of nonalcoholic fatty liver disease (NAFLD) in general Chinese adults. A total of 636 patients with NAFLD and 754 controls from affiliated hospital of Qingdao University in China between January to December 2016 were involved. NAFLD was diagnosed by ultrasonography after excluding other etiologies. The multivariable adjusted odds ratio and $95 \%$ confidence interval (CI) of NAFLD for the highest versus lowest quartile of UHR was 3.888 (2.324-6.504). In stratified analyses by sex and age, the positive associations between UHR and the risk of NAFLD were statistically significant in each subgroup. In stratified by BMI, the significant positive association was only found in the individuals with $\mathrm{BMI} \geq 23.9 \mathrm{~kg} / \mathrm{m}^{2}$. Dose-response analysis indicated a linear positive correlation between UHR and NAFLD risk. 


\section{Introduction}

Nonalcoholic fatty liver disease (NAFLD) is evolved as the major form of the chronic liver disease $(\mathrm{CLD})^{1,2}$ and represents a spectrum of conditions from simple hepatic steatosis to nonalcoholic steatohepatitis (NASH), cirrhosis, and hepatocellular carcinoma ${ }^{3}$. In China, the prevalence of NAFLD is approximately $29.81 \%{ }^{4}$. As hepatic manifestation of metabolic syndrome, NAFLD was closely associated with insulin resistance (IR $)^{5}$, type 2 diabetes(T2DM $)^{6}$, cardiovascular disease ${ }^{7}$ and other chronic diseases ${ }^{8}$. To date, no specific therapy has been approved for treating NAFLD. Therefore, for patients with NAFLD, early screening and prevention are of great importance.

It is well known that NAFLD is closely related to the disorder of lipid metabolism, including highdensity lipoprotein cholesterol (HDL-C) ${ }^{9}$. HDL-C has anti-inflammatory and antioxidant properties and decreased HDL-C concentration is associated with insulin resistance (IR) ${ }^{10}$, the key pathogenesis of NAFLD ${ }^{11}$. Researches by Nemes et al. reported that NAFLD patients usually had low HDL-C levels ${ }^{12-14}$. In addition to lipid abnormalities, serum uric acid (SUA) was also shown to be related to the occurrence and progression of NAFLD ${ }^{15}$. Several studies revealed that populations with higher SUA levels are more likely to develop NAFLD than the general population ${ }^{16-19}$. Recently, studies investigated the associations between the combination of SUA and HDL-C (UHR) and chronic metabolic disease ${ }^{20-22}$. Koncak et al. reported that UHR was a stronger predictor of MS than the other criteria, such as HDL-C, waist circumference and fating plasma glucose ${ }^{20}$. A case-control study conducted by Gulali et al. indicated that UHR could serve as a promising predictor of diabetic control in men with $\mathrm{T}_{2} \mathrm{DM}^{21}$. Besides, a cross-sectional study involving 6285 lean Chinese adults showed a positive association between UHR and NAFLD risk $^{22}$.

To date, evidence on the relationship between UHR and NAFLD risk is limited and no study has explored the dose-response relationship between UHR and NAFLD. Therefore, we conducted this case-control study to explored the association and dose-response relationship of UHR with the risk of NAFLD in general Chinese adults.

\section{Results}

A total of 1390 participants were enrolled (636 NAFLD and 754 non-NAFLD) in this study (Figure1). Comparisons of clinical characteristics of the participants with or without NAFLD are presented in Table 1. Compared with controls, NAFLD patients were more likely to be older, male, current 

smoking and had higher levels of BMI, FBG, ALT, AST, TG, TC, LDL-C, SUA, and UHR, but lower level of HDL-C. The NAFLD subjects also had a higher proportion of diabetes and hypertension than controls.

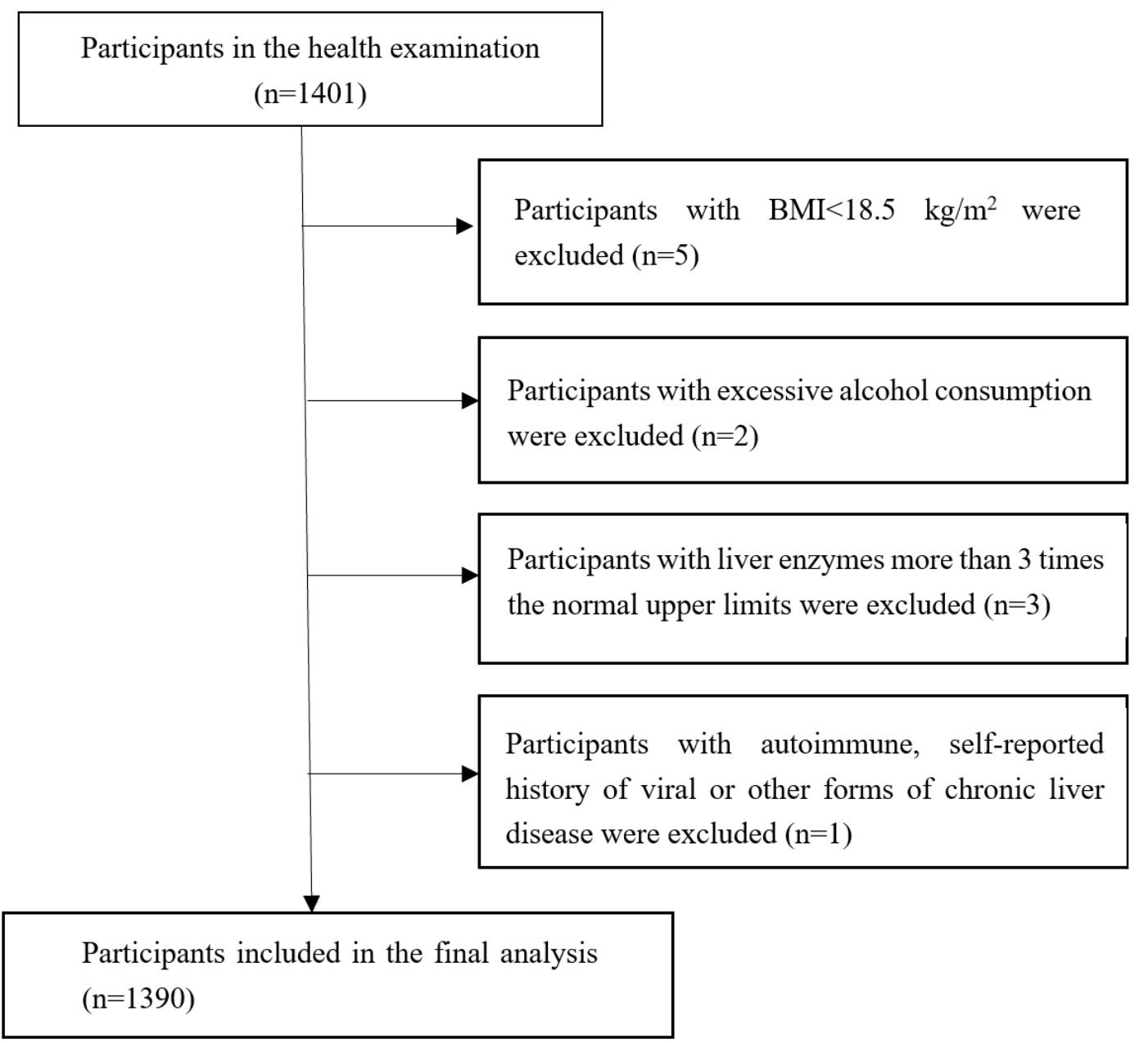

Table 1. Clinical characteristics of participants by NAFLD

\begin{tabular}{llll}
\hline \multirow{2}{*}{ Characteristics } & Non-NAFLD $(\mathbf{n}=\mathbf{7 5 4})$ & NAFLD (n=636) & $\boldsymbol{p}$ value \\
\cline { 2 - 3 } Age, years & Median (IQR) & Median (IQR) & 0.004 \\
BMI, $\mathrm{kg} / \mathrm{m}^{2}$ & $50(44,57)$ & $52(45,58)$ & $<0.001$ \\
ALT, U/L & $23.78(22.03,25.60)$ & $26.59(24.97,28.65)$ & $<0.001$ \\
AST, U/L & $18(14,24)$ & $24(19,36)$ & $<0.001$ \\
FPG, $\mathrm{mmol} / \mathrm{L}$ & $19(16,21)$ & $20(17,24)$ & $<0.001$ \\
TG, $\mathrm{mg} / \mathrm{dL}$ & $5.0(4.8,5.4)$ & $5.4(5.0,6.0)$ & $<0.001$ \\
TC, $\mathrm{mg} / \mathrm{dL}$ & $74.4(53.2,108.1)$ & $128.0(90.4,187.8)$ & $208.6(185.1,238.8)$
\end{tabular}




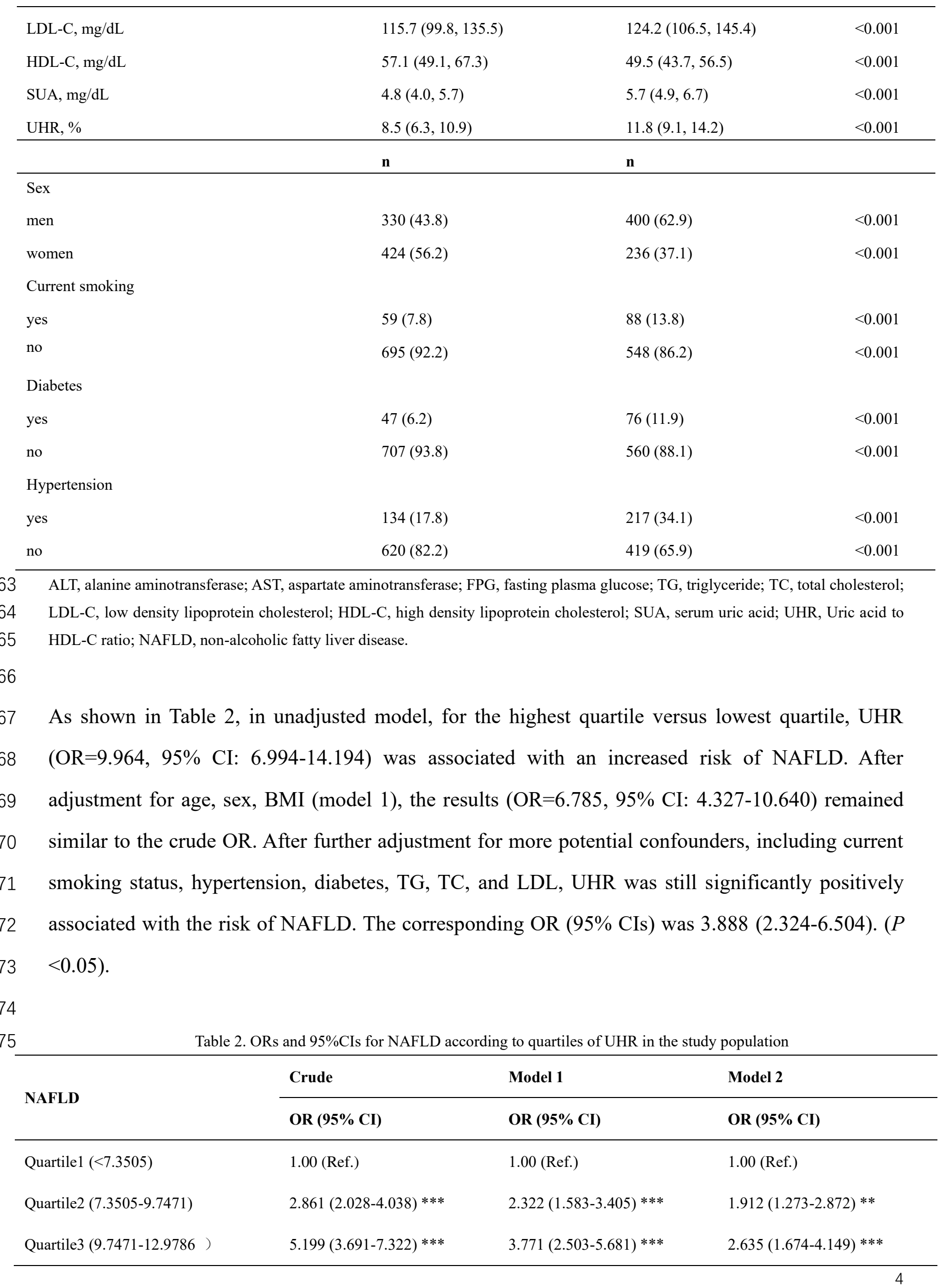


${ }^{*} p<0.05,{ }^{* *} p<0.01, * * * p<0.001$.

The relationships between UHR and NAFLD risk in different subgroups were presented in Table 3, Table 4 and Table 5, respectively. In stratified analyses by sex, compared with the lowest quartile, the multivariate ORs (95\% CIs) of NAFLD for the highest quartile of UHR for men and women were 2.374 (1.344-4.196), 3.011 (1.538-5.894), respectively. In stratified analyses by age, for participants younger than 50 years old, the OR $(95 \% \mathrm{CI})$ of NAFLD for the highest quartile vs. lowest quartile of UHR was 7.534 (2.916-19.465) in multivariate analysis. The OR (95\% CI) was 3.063 (1.642-5.714) for subjects aged $50^{+}$years. Analysis stratified by BMI indicated that the association was more pronounced in participants with BMI $\geq 23.9 \mathrm{~kg} / \mathrm{m}^{2}$ and the ORs (95\% CIs) of NAFLD were 1.442 $(0.948-2.196)$ in quartile 2, $2.370(1.447-3.883)$ in quartile 3 , and 2.940 (1.685-5.130) in quartile 4 (model 2). For participants with $18.5 \leq \mathrm{BMI}<23.9 \mathrm{~kg} / \mathrm{m}^{2}$, no significant association was observed between UHR and NAFLD.

92

Table 3. ORs and $95 \%$ CIs for NAFLD according to quartiles of UHR in the study population, stratified by sex

\begin{tabular}{|c|c|c|c|}
\hline \multirow{2}{*}{ NAFLD } & Crude & Model 1 & Model 2 \\
\hline & OR $(95 \% \mathrm{CI})$ & OR $(95 \% \mathrm{CI})$ & OR $(95 \% \mathrm{CI})$ \\
\hline \multicolumn{4}{|l|}{ men } \\
\hline \multicolumn{4}{|l|}{ UHR quartile } \\
\hline Quartile1 (<9.7656) & 1.00 (Ref.) & 1.00 (Ref.) & 1.00 (Ref.) \\
\hline Quartile2 (9.7656-12.2748) & $1.788(1.174-2.725)^{* *}$ & $1.354(0.849-2.159)$ & $1.169(0.711-1.922)$ \\
\hline Quartile3 (12.2748-14.6127) & $2.980(1.946-4.563) * * *$ & $2.065(1.293-3.298) * *$ & $1.598(0.956-2.672)$ \\
\hline Quartile $4(\geq 14.6127)$ & $5.585(3.551-8.783) * * *$ & $3.301(2.007-5.432) * * *$ & $2.374(1.344-4.196) * *$ \\
\hline \multicolumn{4}{|l|}{ women } \\
\hline \multicolumn{4}{|l|}{ UHR quartile } \\
\hline Quartile $1(<5.8793)$ & 1.00 (Ref.) & 1.00 (Ref.) & 1.00 (Ref.) \\
\hline Quartile 2 (5.8793-7.6255) & $1.376(0.789-2.400)$ & $1.019(0.559-1.856)$ & $0.836(0.439-1.595)$ \\
\hline Quartile3 (7.6255-9.4763) & $3.583(2.139-6.002)^{* * *}$ & $2.469(1.411-4.319) * *$ & $1.614(0.855-3.045)$ \\
\hline Quartile $4(\geq 9.4763)$ & $9.183(5.454-15.461) * * *$ & $5.799(3.305-10.176) * * *$ & $3.011(1.538-5.894) * * *$ \\
\hline
\end{tabular}




\begin{tabular}{|c|c|c|c|}
\hline \multirow{2}{*}{ NAFLD } & Crude & Model 1 & Model 2 \\
\hline & OR $(95 \%$ CI $)$ & OR $(95 \%$ CI $)$ & OR $(95 \%$ CI $)$ \\
\hline \multicolumn{4}{|l|}{$<50$ years } \\
\hline \multicolumn{4}{|l|}{ UHR quartile } \\
\hline Quartile1 $(<7.0953)$ & 1.00 (Ref.) & 1.00 (Ref.) & 1.00 (Ref.) \\
\hline Quartile2 (7.0953-9.6465) & $4.951(2.601-9.426) * * *$ & $3.500(1.713-7.148) * *$ & $3.294(1.532-7.086) * *$ \\
\hline Quartile3 (9.6465-13.2329) & $10.000(5.306-18.846) * * *$ & $5.694(2.678-12.104) * * *$ & $4.332(1.868-10.045) * * *$ \\
\hline Quartile 4 ( $\geq 13.2329)$ & $28.500(14.775-54.973) * * *$ & $11.169(4.863-25.655) * * *$ & $7.534(2.916-19.465) * * *$ \\
\hline \multicolumn{4}{|l|}{$\geq$ 50years } \\
\hline \multicolumn{4}{|l|}{ UHR quartile } \\
\hline Quartile1 $(<7.4813)$ & 1.00 (Ref.) & 1.00 (Ref.) & 1.00 (Ref.) \\
\hline Quartile2 (7.4813-9.7943) & $2.148(1.405-3.283) * * *$ & $1.993(1.249-3.180) * *$ & $1.589(0.968-2.608)$ \\
\hline Quartile3 (9.7943-12.7691) & $3.459(2.262-5.291) * * *$ & $3.113(1.891-5.124) * * *$ & $2.113(1.215-3.673) * *$ \\
\hline Quartile $4(\geq 12.7691)$ & $5.451(3.523-8.433) * * *$ & $5.374(3.131-9.224) * * *$ & $3.063(1.642-5.714) * * *$ \\
\hline
\end{tabular}

$100 \quad$ CI, confidence interval; OR, odds ratio.

101 Model 1 Adjusted for sex, BMI.

102 Model 2 Adjusted for sex, BMI, current smoking, diabetes, hypertension, TG, TC, LDL.

$103 * p<0.05, * * p<0.01, * * * p<0.001$.

104

105

Table 5. ORs and 95\%CIs for NAFLD according to quartiles of UHR in the study population, stratified by BMI

\begin{tabular}{llll}
\hline \multirow{2}{*}{ NAFLD } & Crude & Model 1 & Model 2 \\
\cline { 2 - 3 } & OR (95\% CI) & OR (95\% CI) & OR (95\% CI) \\
\hline
\end{tabular}

\section{$18.5 \leq \mathrm{BMI}<23.9 \mathrm{Kg} / \mathrm{m}^{2}$}

\section{UHR quartile}

Quartile1 $(<5.9387)$

Quartile2 (5.9387-7.9646)

Quartile3 (7.9646-10.0310)

Quartile4 ( $\geq 10.0310)$

$B M I \geq 23.9 \mathrm{Kg} / \mathrm{m}^{2}$

\section{UHR quartile}

Quartile1 $(<8.4319)$

$$
1.00 \text { (Ref.) }
$$

Quartile2 (8.4319 -11.0328)

$1.843(1.274-2.667) * *$

Quartile3 (11.0328-13.7509)

Quartile4 ( $\geq 13.7509)$

1.00 (Ref.)

$0.810(0.335-1.956)$

$1.680(0.775-3.642)$

3.302(1.602-6.804) **
1.00 (Ref.)

$0.705(0.283-1.754)$

1.555 (0.681-3.553)

$4.039(1.724-9.460) * *$
1.00 (Ref.)

$0.685(0.257-1.822)$

$1.273(0.504-3.215)$

$2.463(0.883-6.870)$

106 CI, confidence interval; OR, odds ratio.

107 Model 1 Adjusted for age, sex. 
$109 * p<0.05, * * p<0.01, * * * p<0.001$.

The dose-response association of UHR with NAFLD risk in the restricted cubic spline model was presented in Figure 2. UHR was linearly positively related to the risk of NAFLD ( $p$ for nonlinearity $=0.193)$. When the UHR index was 5, the OR value tended to be the lowest (OR:1.22;95\% CI:1.081.37).

Figure2. Dose-response relationship between UHR and the risk of NAFLD. Adjustments were made according to age, sex, BMI, current smoking, diabetes, hypertension, TG, TC, LDL. The solid line and the dotted line represent the estimated OR and the corresponding $95 \% \mathrm{CI}$, respectively. OR, odds ratio.

\section{Discussion}

In this case-control study, we observed a positive association between UHR and NAFLD risk in Chinese adults, after adjustment for multiple potential confounders (age, sex, BMI, current smoking, diabetes, hypertension, TG, TC, LDL-C). In stratified analysis by sex and age, the positive correlations between UHR and the risk of NAFLD were significant in subgroups with different sex and age, while in stratified analysis by BMI, positive association was only observed in participants with $\mathrm{BMI} \geq 23.9 \mathrm{~kg} / \mathrm{m}^{2}$ after adjustment for confounding factors. Furthermore, a linear positive association between UHR and the risk of NAFLD was observed. To our knowledge, this is the first time to explore the dose-response relationship between UHR and NAFLD risk. SUA and HDL-C are considered as the two crucial metabolic variables altered in fatty liver. As an end product of purine metabolism, elevated SUA concentration increased the risk of NAFLD ${ }^{23}$ and was considered as an independent risk factor for the development of NAFLD and aggravation of liver damage in population ${ }^{24-26}$. HDL-C is mainly synthesized in the liver, decreased HDL-C levels were 
caused by lacking of exercise ${ }^{27}$, smoking $^{28}$, obesity ${ }^{29}$ and diabetes ${ }^{30}$, which are risk factors of $\mathrm{NAFLD}^{31,32}$. In fact, patients with NAFLD often have lower HDL-C level. Recently, there are several studies reported that the ratio of SUA and HDL-C (UHR) was closely related to metabolic diseases. Kocak et al. demonstrated that UHR was a better predictor than other established criterion of MS in a case-control study with 100 type 2 diabetic subjects ${ }^{20}$. Research conducted in 159 men with T2DM showed that UHR is a promising index in predicting of diabetic control ${ }^{21}$. In a cross-sectional study, UHR was found to be significantly associated with NAFLD in 6285 lean Chinese subjects ${ }^{22}$. Our finding of the positive association between UHR and the risk of NAFLD was similar to the aforementioned studies. To our knowledge, this is the first study to explore the relationship between UHR and NAFLD, stratified by gender, age and BMI. As routine detection variables in clinical laboratories, the ratio of SUA to HDL-C (UHR) can serve as a reliable and non-invasive marker for predicting NAFLD in Chinese adults.

Our study has some strengths. First, the relatively large sample size increased the statistical power and reliability of the results. Second, we conducted stratification analysis to better understand the association between UHR and NAFLD risk in different subgroup of the study population. Third, the positive association of UHR with NAFLD risk remained statistically significant after adjustment for potential confounders. There are also several limitations in our study. First, this study was a casecontrol design, the causal association between UHR and NAFLD could not be precisely identified. In the future, a long-term cohort study in larger population is required. Secondly, although ultrasound scan has a good sensitivity and specificity in identifying fatty liver, it is not the gold standard for NAFLD diagnosis. Third, there may be residual confusions caused by incomplete adjustment.

In conclusion, UHR is positively associated with NAFLD, and may serve as an innovative and noninvasive marker in identifying individuals at risk for NAFLD in Chinese adults.

\section{Methods}

\section{Study population}

This study is a case-control design focused on Chinese Han population aged 20 70 years. Subjects were recruited from Medical Examination Center of the Affiliated Hospital of Qingdao University from January to December 2016. Questionnaire survey, abdominal ultrasound examination and blood biochemical tests were performed in all participants for the diagnosis of NAFLD. Those who had any of the following behaviors or symptoms were excluded: (i) $\mathrm{BMI}<18.5 \mathrm{~kg} / \mathrm{m}^{2}$; ${ }^{4}$ excessive alcohol drinking (>140 g/week for men and $>70 \mathrm{~g} /$ week for women); (iii) other unexplained elevated liver enzymes or transaminases 3 times higher than the upper limit of normal (laboratory normal range: 0 
$39 \mathrm{U} / \mathrm{L})$; the presence of autoimmune, self-reported history of viral, or other forms of chronic liver disease. The healthy control samples were derived from the same center during the same study period (Figure 1). The study was approved by the Ethical Committee of Medical College of Qingdao University (Ethical approval number: [Medical College of Qingdao University 20130304]; Clinical trial registration number: ChiCTR-OCS-14004819).

\section{Data collection and measurements}

All the participants took a complete physical examination in the morning after a 12-hour overnight fast. Standardized questionnaires were used to collect information of age, gender, smoking and alcohol consumption. Alcohol consumption was assessed according to the frequency of alcohol intake per week and the usual amount of alcohol consumed per occasion. Height and body weight were measured using standardized procedures. Body mass index (BMI) was calculated as body weight $(\mathrm{kg}) /[\text { height }(\mathrm{m})]^{2}$, and classified into two categories: normal weight $18.5 \leq \mathrm{BMI}<23.9 \mathrm{~kg} / \mathrm{m}^{2}$; overweight or obese BMI $\geq 23.9 \mathrm{~kg} / \mathrm{m}^{2}$. Systolic and diastolic blood pressures were measured using a standard mercurial sphygmomanometer after a 10-minute rest in the sitting position. Overnight fasted blood samples were obtained for the analysis of biochemical variables including serum aspartate aminotransferase (AST), alanine aminotransferase (ALT), SUA, serum fasting blood glucose (FBG), total cholesterol, triglycerides, low-density lipoprotein cholesterol (LDL-C) and high-density lipoprotein cholesterol (HDL-C), which were measured by an automatic analyzer (Beckman CX-7 Biochemical Autoanalyzer, Brea, CA, USA).

\section{Definitions}

Hepatic steatosis was diagnosed according to abdominal ultrasound results by trained technicians. The ultrasonic diagnosis of fatty liver was based on the criteria proposed by the Chinese Society of Endocrinology ${ }^{33}$. Diabetes Mellitus was defined as $\mathrm{FBG} \geq 7.0 \mathrm{mmol} / \mathrm{L}$, or self-reported diabetes diagnosis, or current use of anti-diabetes treatment ${ }^{34}$. Hypertension was defined as: systolic blood pressure $\geq 140 \mathrm{mmHg}$ and /or diastolic blood pressure $\geq 90 \mathrm{mmHg}$, or current treatment for hypertension or a history of hypertension ${ }^{35}$.

\section{Statistical analysis}

Characteristics of the subjects were presented as median and quartiles for categorical variables. Mann Whitney U tests was used to evaluate the differences between participants with and without NAFLD. UHR was categorized based on quartiles (quartile $1:<25$ th percentile, quartile $2: \geq 25$ th to 50 th percentile, quartile $3: \geq 50$ th to 75 th percentile, quartile $4: \geq 75$ th percentile). The odds ratio (OR) with 95\% confidence intervals (CIs) were calculated from binary logistic regression analyses to determine the association of UHR with the risk of NAFLD. In binary logistic regression analyses, model 1 was adjusted for age, sex and BMI. Model 2 was adjusted for age, sex, BMI, current smoking, diabetes, 
199 hypertension, TG, TC and LDL-C. Stratified analyses were performed based on age ( $<50$ y and $\geq 50$ y), sex (men and women), and BMI $\left(18.5 \leq \mathrm{BMI}<23.9 \mathrm{~kg} / \mathrm{m}^{2}\right.$ and $\left.\mathrm{BMI} \geq 23.9 \mathrm{~kg} / \mathrm{m}^{2}\right)$ to evaluate the association between UHR and NAFLD risk. Dose-response relationships were evaluated using a restricted cubic spline function with three knots located at the 5, 50, and 95th percentiles of the exposure distribution in the fully adjusted model. The non-linear p-value was calculated by testing the value of the quadratic zero spline coefficient. Statistical analyses were carried out with Stata.V.15.0. A two-tailed $p$-value $<0.05$ indicated statistically significant.

\section{References}

1 Younossi, Z. M. Non-alcoholic fatty liver disease - A global public health perspective. J Hepato/ 70, 531544, (2019).

2 Maurice, J. \& Manousou, P. Non-alcoholic fatty liver disease. Clinical medicine (London, England) 18, 245250, (2018).

3 George, J., Anstee, Q., Ratziu, V. \& Sanyal, A. NAFLD: The evolving landscape. J Hepato/ 68, 227-229, (2018).

$4 \quad \mathrm{Li}$, J. et al. Prevalence, incidence, and outcome of non-alcoholic fatty liver disease in Asia, 1999-2019: a systematic review and meta-analysis. The Lancet Gastroenterology \& Hepatology 4, 389-398, (2019).

$5 \quad$ Khan, R. S., Bril, F., Cusi, K. \& Newsome, P. N. Modulation of Insulin Resistance in Nonalcoholic Fatty Liver Disease. Hepatology 70, 711-724, (2019).

6 Fukuda, T. et al. The impact of non-alcoholic fatty liver disease on incident type 2 diabetes mellitus in non-overweight individuals. Liver international : official journal of the International Association for the Study of the Liver 36, 275-283, (2016).

7 Stepanova, M. \& Younossi, Z. M. Independent association between nonalcoholic fatty liver disease and cardiovascular disease in the US population. Clinical gastroenterology and hepatology : the official clinical practice journal of the American Gastroenterological Association 10, 646-650, (2012). Marcuccilli, M. \& Chonchol, M. NAFLD and Chronic Kidney Disease. Int J Mol Sci 17, 562, (2016). Speliotes, E. K. et al. Fatty liver is associated with dyslipidemia and dysglycemia independent of visceral fat: the Framingham Heart Study. Hepatology 51, 1979-1987, (2010).

Ren, X. et al. Association between Triglyceride to HDL-C Ratio (TG/HDL-C) and Insulin Resistance in Chinese Patients with Newly Diagnosed Type 2 Diabetes Mellitus. PloS one 11, e0154345, (2016).

Watt, M. J., Miotto, P. M., De Nardo, W. \& Montgomery, M. K. The Liver as an Endocrine Organ-Linking NAFLD and Insulin Resistance. Endocrine reviews 40, 1367-1393, (2019).

Nemes, K. \& Åberg, F. Interpreting lipoproteins in nonalcoholic fatty liver disease. Current opinion in lipidology 28, 355-360, (2017).

$13 \mathrm{Wu}, \mathrm{K} . \mathrm{T}$. et al. Nonalcoholic fatty liver disease severity is associated with the ratios of total cholesterol and triglycerides to high-density lipoprotein cholesterol. Journal of clinical lipidology 10, 420-425.e421, (2016).

14 DeFilippis, A. P. et al. Nonalcoholic fatty liver disease and serum lipoproteins: the Multi-Ethnic Study of Atherosclerosis. Atherosclerosis 227, 429-436, (2013).

15 Ma, Z. et al. Changing trajectories of serum uric acid and risk of non-alcoholic fatty liver disease: a prospective cohort study. J Trans/ Med 18, 133, (2020).

Jaruvongvanich, V., Ahuja, W., Wirunsawanya, K., Wijarnpreecha, K. \& Ungprasert, P. Hyperuricemia is associated with nonalcoholic fatty liver disease activity score in patients with nonalcoholic fatty liver 
disease: a systematic review and meta-analysis. Eur J Gastroenterol Hepato/ 29, 1031-1035, (2017).

Wei, F. et al. Higher Serum Uric Acid Level Predicts Non-alcoholic Fatty Liver Disease: A 4-Year Prospective Cohort Study. Frontiers in Endocrinology 11, (2020).

Zheng, X. et al. Serum uric acid and non-alcoholic fatty liver disease in non-obesity Chinese adults. Lipids Health Dis 16, 202, (2017).

Liu, C. Q. et al. Serum uric acid is independently and linearly associated with risk of nonalcoholic fatty liver disease in obese Chinese adults. Sci Rep 6, 38605, (2016).

Kocak, M. Z. et al. Serum uric acid to HDL-cholesterol ratio is a strong predictor of metabolic syndrome in type 2 diabetes mellitus. Revista da Associacao Medica Brasileira (1992) 65, 9-15, (2019).

Aktas, G. et al. Uric acid to HDL cholesterol ratio is a strong predictor of diabetic control in men with type 2 diabetes mellitus. The aging male : the official journal of the International Society for the Study of the Aging Male 23, 1098-1102, (2020).

Zhang, Y. N., Wang, Q. Q., Chen, Y. S., Shen, C. \& Xu, C. F. Association between Serum Uric Acid to HDLCholesterol Ratio and Nonalcoholic Fatty Liver Disease in Lean Chinese Adults. International journal of endocrinology 2020, 5953461, (2020).

$\mathrm{Xu}, \mathrm{C}$. Hyperuricemia and nonalcoholic fatty liver disease: from bedside to bench and back. Hepatology international 10, 286-293, (2016). Liver Disease: A Meta-Analysis. Acta medica Indonesiana 49, 136-147, (2017).

Liu, Z., Que, S., Zhou, L. \& Zheng, S. Dose-response Relationship of Serum Uric Acid with Metabolic Syndrome and Non-alcoholic Fatty Liver Disease Incidence: A Meta-analysis of Prospective Studies. Scientific reports 5, 14325, (2015).

Zhou, Y., Wei, F. \& Fan, Y. High serum uric acid and risk of nonalcoholic fatty liver disease: A systematic review and meta-analysis. Clinical biochemistry 49, 636-642, (2016).

Maylor, B. D., Zakrzewski-Fruer, J. K., Orton, C. J. \& Bailey, D. P. Beneficial postprandial lipaemic effects of interrupting sedentary time with high-intensity physical activity versus a continuous moderate-intensity physical activity bout: A randomised crossover trial. Journal of science and medicine in sport 21, 12501255, (2018).

Chen, H. Y. et al. The effects of cigarette smoking and smoking cessation on high-density lipoprotein functions: implications for coronary artery disease. Annals of clinical biochemistry 56, 100-111, (2019).

Escalante, Y., Saavedra, J. M., García-Hermoso, A. \& Domínguez, A. M. Improvement of the lipid profile with exercise in obese children: a systematic review. Preventive medicine 54, 293-301, (2012).

Waldman, B. et al. HDL-C and HDL-C/ApoA-I predict long-term progression of glycemia in established type 2 diabetes. Diabetes care 37, 2351-2358, (2014).

Zhang, Q. Q. \& Lu, L. G. Nonalcoholic Fatty Liver Disease: Dyslipidemia, Risk for Cardiovascular Complications, and Treatment Strategy. Journal of clinical and translational hepatology 3, 78-84, (2015). Chatrath, H., Vuppalanchi, R. \& Chalasani, N. Dyslipidemia in patients with nonalcoholic fatty liver disease. Seminars in liver disease 32, 22-29, (2012).

Gao, X. \& Fan, J. G. Diagnosis and management of non-alcoholic fatty liver disease and related metabolic disorders: consensus statement from the Study Group of Liver and Metabolism, Chinese Society of Endocrinology. Journal of diabetes 5, 406-415, (2013). Petersmann, A. et al. Definition, Classification and Diagnosis of Diabetes Mellitus. 127, S1-S7, (2019). 2018 Chinese Guidelines for Prevention and Treatment of Hypertension-A report of the Revision Committee of Chinese Guidelines for Prevention and Treatment of Hypertension. Journal of geriatric cardiology : JGC 16, 182-241, (2019). 


\section{Acknowledgements}

288 The authors thank all of the people who participated in this study. This study was supported by the 289 Natural Science Foundation of China [No. 81703206, No. 81973015] and Science and Technology 290 Program of Qingdao [No. 19-6-1-52-nsh].

\section{Author Contributions}

Yongye Sun designed the study. Xia Qiu and Jiajia Cui acquired the data. Hui Zhao analyzed the data 293 and drafted the manuscript. Yongye Sun critically revised the manuscript. The work was carried out at the Qingdao University. All authors read and approved the final manuscript.

\section{Additional Information}

296 Competing interests

297 The authors declare no competing interests.

298 Ethical Standards Disclosure: This study was conducted according to the guidelines laid down in 299 the Declaration of Helsinki and all procedures involving research study participants were approved by the Ethical Committee of Medical College of Qingdao University. Written informed consent was 301 obtained from all subjects. 